
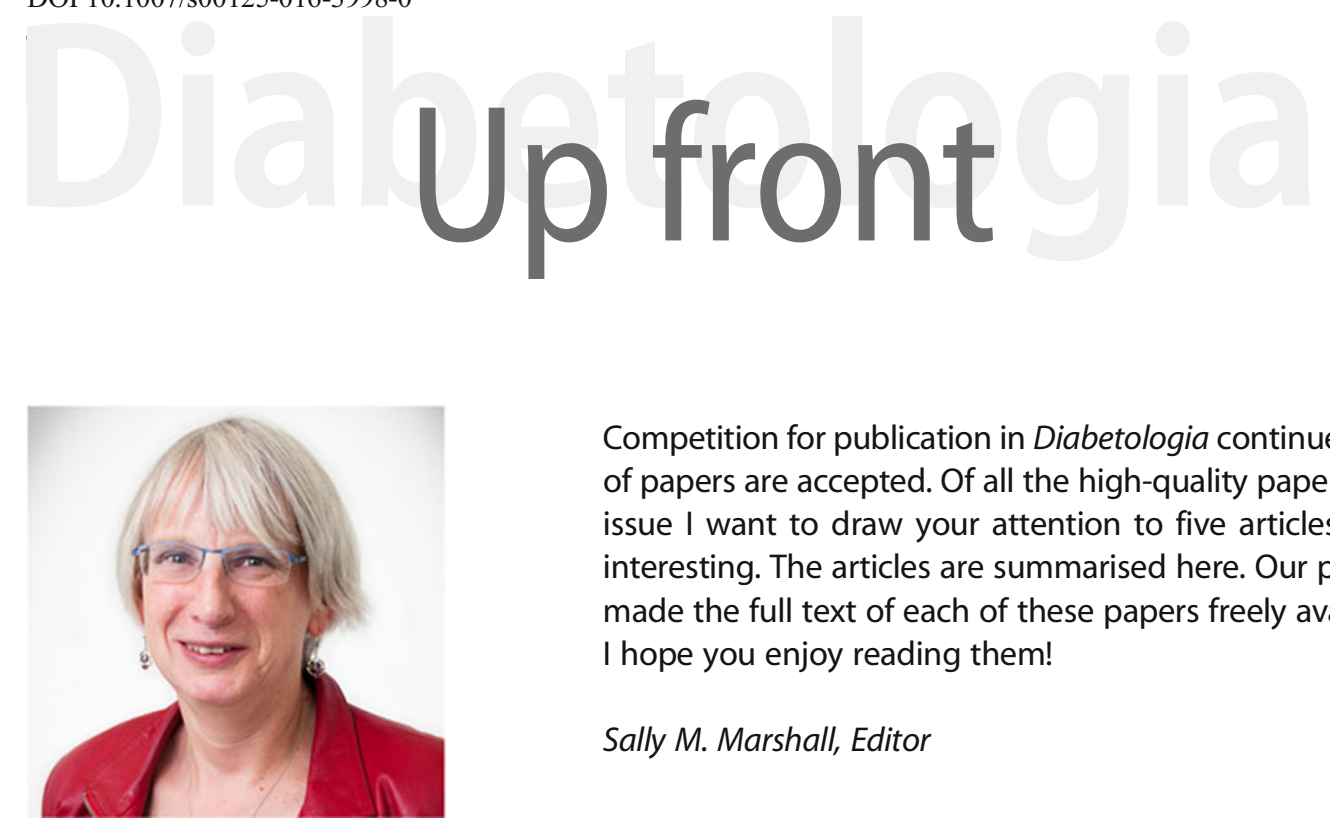

Competition for publication in Diabetologia continues to grow, and less than 20\% of papers are accepted. Of all the high-quality papers that appear in this month's issue I want to draw your attention to five articles that I think are particularly interesting. The articles are summarised here. Our publisher, Springer, has kindly made the full text of each of these papers freely available.

I hope you enjoy reading them!

Sally M. Marshall, Editor

\section{SGLT2 Inhibition and cardiovascular events: why did EMPA-REG Outcomes surprise and what were the likely mechanisms?}

Naveed Sattar, James McLaren, Søren L. Kristensen, David Preiss, John J. McMurray

The $30-40 \%$ reductions in hospitalisation for heart failure, cardiovascular mortality and total mortality yielded by empagliflozin treatment in the placebo-controlled EMPA-REG Outcomes trial took the research community by surprise. This pattern of benefit and its rapid emergence, along with the lack of clear reductions in myocardial infarction or stroke, point towards non-atherothrombotic mechanisms. In a review in this issue, Sattar et al argue that the beneficial effects of empagliflozin are likely to stem from its corrective actions on the abnormal renal glucose and sodium handling, along with the intraglomerular hypertension, which occur in type 2 diabetes. The authors explain how these actions reduce the development and progression of heart failure via improvements in cardiac function and speculate which other groups of patients may benefit from the sodium-glucose linked transporter-2 (SGLT2) inhibitor class of glucoselowering therapy. Finally, the authors suggest that the EMPA-REG Outcome trial findings highlight our limited appreciation of the marked risk for developing heart failure in many patients with type 2 diabetes.
In vivo imaging of beta cells with radiotracers: state of the art, prospects and recommendations for development and use

Olof Eriksson, Maren Laughlin, Maarten Brom, Pirjo Nuutila, Michael Roden, Albert Hwa, Riccardo Bonadonna, Martin Gotthardt

Beta cell dysfunction and death are presumed to be central events in the pathogenesis of type 1 and type 2 diabetes in humans, but until recently there have been few direct observations regarding the fate of beta cells during the course of disease. In this issue, Eriksson and colleagues review recent advances in beta cell imaging by radiolabelled ligands (radiotracers). During the last decade, the non-invasive imaging technologies positron emission tomography (PET) and single photon emission computed tomography (SPECT) have been applied to the direct in situ radiolabelling of beta cells, potentially permitting repeated measurements of beta cell mass in humans. Preclinical and clinical data indicate that the scientific community now has access to several radiolabelled ligands with variable but high specificity for human beta cells. Further clinical validation of these imaging biomarkers may improve our understanding of the natural history of the human beta cell and its fate in diabetes. 


\section{Islet-intrinsic effects of CFTR mutation}

Fiona N. Manderson Koivula, Neville H. McClenaghan, Alan G. S. Harper, Catriona Kelly

Cystic fibrosis (CF) is primarily associated with lung disease, which is the main cause of morbidity and mortality in this genetic condition and arises as a result of chronic/recurring lung infections. However, diabetes is the most common extra-pulmonary comorbidity and presents significant problems to those with $C F$, since hyperglycaemia accelerates microbial colonisation and lung decline. In a review in this issue, Manderson Koivula et al present emerging evidence that mutations in the gene encoding cystic fibrosis transmembrane conductance regulator (CFTR) that are responsible for CF development cause significant dysregulation of islet cell function, leading to altered glucose homeostasis and insulin secretion. The authors discuss recent research findings that support the view that CFTR deficiency or mutation has a negative impact on beta and islet cell function, highlighting the need for an enhanced understanding of the cellular mechanisms underlying the clinical development of CF-related diabetes in order to better treat the disease.

\section{Burden of diabetes in Australia: life expectancy and disability-free life expectancy in adults with diabetes}

Lili Huo, Jonathan E. Shaw, Evelyn Wong, Jessica L. Harding, Anna Peeters, Dianna J. Magliano

The rising prevalence of diabetes, together with improvements in the life expectancy of individuals with this condition, has prompted a greater emphasis to be placed on health-related quality of life. In this issue, using Australian national datasets for mortality (2011) and disability prevalence (2012), Huo et al report that the estimated loss of life expectancy of individuals with diabetes at age 50 years was approximately 3 years compared with their counterparts without diabetes. However, the corresponding estimated loss of disability- free life expectancy (DFLE) was much greater, at 8.2 years for men and 9.1 years for women. Women with diabetes spend more absolute years, and a larger proportion of their life with disability compared with men with diabetes and women without diabetes. The loss of DFLE in people with diabetes is likely to be multifactorial, and may include physical and cognitive impairment as well as traditional diabetes complications. Moving forward, efforts to identify the specific causes of disability may potentiate effective interventions for loss of DFLE in diabetes.

\section{Evaluation of type 2 diabetes genetic risk variants in Chinese adults: findings from 93,000 individuals from the China Kadoorie Biobank}

Wei Gan, Robin G. Walters, Michael V. Holmes, Fiona Bragg, Iona Y. Millwood, Karina Banasik, Yiping Chen, Huaidong Du, Andri lona, Anubha Mahajan, Ling Yang, Zheng Bian, Yu Guo, Robert J. Clarke, Liming Li, Mark I. McCarthy, Zhengming Chen, on behalf of the China Kadoorie Biobank Collaborative Group

Over the past decade, researchers have used large-scale genetic studies to identify over 90 regions of the genome at which DNA sequence differences influence the risk that an individual will develop type 2 diabetes. However, most of these studies have been conducted in European individuals, and the designs used may have overestimated the size of these genetic effects. In this issue, Gan, Walters and colleagues report on the impact of 56 known diabetes risk variants on predisposition to type 2 diabetes in a prospective cohort study of 93,000 Chinese adults. Although many of these signals were first detected in Europeans, the results of this study demonstrate that most also influence diabetes risk in the Chinese population, albeit with somewhat lesser effects. These data establish shared patterns of genetic risk factors for diabetes in ethnically distinct populations, and will support ongoing research efforts to map the pathways responsible for diabetes development. This article is the subject of a commentary in this issue by Marco Dauriz and James Meigs.

All text supplied by the authors. 